\title{
PROMOVER MODELOS EXPLICATIVOS SOBRE LAS INTERACCIONES QUÍMICAS DEL FELODIPINO- CITOCROMO P450: UNA PROPUESTA DIDÁCTICA BASADA EN LA MODELIZACIÓN
}

\author{
Rafael Amador-Rodríguez ${ }^{\mathrm{a}, *,(1)}$, Daniel Insuasty ${ }^{\mathrm{b}}$, Maximiliano Méndez-López $^{\mathrm{b}}$ y Edgar Márquez ${ }^{\mathrm{b}}$ \\ ${ }^{a}$ Grupo Cognición y Educación, Instituto de Estudios en Educación, Universidad del Norte, km 5 vía puerto Colombia, Barranquilla, \\ Colombia \\ ${ }^{\text {b} G r u p o ~ d e ~ Q u i ́ m i c a ~ y ~ B i o l o g i ́ a, ~ D e p a r t a m e n t o ~ d e ~ Q u i ́ m i c a ~ y ~ B i o l o g i ́ a, ~ U n i v e r s i d a d ~ d e l ~ N o r t e, ~ k m ~} 5$ vía puerto Colombia, \\ Barranquilla, Colombia
}

Recebido em 23/02/2021; aceito em 12/05/2021; publicado na web em 16/06/2021

\begin{abstract}
PROMOTING EXPLANATORY MODELS ON FELODIPINE-CYTOCHROME P450 INTERACTION: A DIDACTIC PROPOSAL MODELING-BASED. This article presents the results of the study of explanatory models produced by 81 students of first-year of medicine at the Universidad del Norte in Barranquilla-Colombia. The students face a case in the area of chemistry applied to medicine, through a contextualized problem situation (SPC), constructed from a perspective of teaching chemistry in context. The explanatory models generated by the students were analyzed qualitatively at four moments of the implementation of a teaching proposal. The results obtained at the beginning of the implementation of the proposal indicate that the students did not elaborate any type of explanatory model. However, these arised from the teaching proposal that involves the SPC in which the students begin to generate explanatory models supported by chemistry content and categorized mostly in the research as Descriptive and Interpretative, a situation fostered by the theoretical and methodological coherence of the SPC, which was designed from a perspective of teaching in context based on modeling and leveraged by scaffolding.
\end{abstract}

Keywords: modeling; scaffolding; teach in context; explanatory models and teaching chemistry.

\section{INTRODUCCIÓN}

En los últimos años se ha incrementado en el campo de la didáctica de las ciencias naturales el interés por estudiar cómo se desarrollan o evolucionan las ideas de los estudiantes acerca de conceptos científicos relevantes según el nivel educativo. ${ }^{1}$ Uno de los problemas que se ha determinado en el aprendizaje de la química es que los estudiantes regularmente acogen una visión realista ingenua sobre el mundo natural, y la utilizan para formular modelos explicativos de lo que sucede en el nivel microscópico de la química (átomos, moléculas, redes iónicas, interacciones, etc.). Estos modelos explicativos surgen ya que los estudiantes consideran que los fenómenos que acontecen en el mundo micro se comportan igual que en el mundo macro, sin comprender que existen distintos niveles de representación de los materiales en constante relación. Por ejemplo, a un medicamento se le puede determinar sus propiedades física y químicas a nivel macroscópico, sin embargo, para lograr explicar su efectividad química y biológica es necesario entender su comportamiento a nivel microscópico, lo que se puede lograr utilizando simulaciones computacionales que permiten estudiar las interacciones fármaco-receptor. ${ }^{2}$

Como una solución al problema en la construcción de modelos explicativos de los fenómenos químicos por parte de los estudiantes, se ha propuesto como alternativa una enseñanza de la química en contexto basadas en la modelización. ${ }^{3}$ Actualmente, se ha planteado que los profesores de ciencias naturales enseñen modelos y teorías vigentes, que surgen de situaciones problemáticas abordadas por los científicos, las cuales requieren de una situación real en la que se representa, interpreta y explica un fenómeno, otorgándole sentido a la construcción de conocimiento científico, por ejemplo, los químicos han propuesto modelos para simular los distintos materiales y así poder describir, explicar y predecir las propiedades

*e-mail: ryamador@uninorte.edu.co y los comportamientos de los mismos. ${ }^{4}$ Algunos de estos modelos y teorías hacen parte de los planes curriculares para la formación de profesionales en áreas específicas, así como en la formación de médicos que se propone enseñar enlace químico, termoquímica y química orgánica como temáticas que contribuyan a dicha formación.

\section{Modelización: Operar con modelos en el aula}

Algunos investigadores en didáctica de las ciencias acuden a los planteamientos epistemológicos de Ronald Giere ${ }^{5}$ por su plausibilidad para entender la construcción del conocimiento científico escolar. ${ }^{6-10}$ Una de las categorías epistemológicas que se ha interpretado en la didáctica de las ciencias es la de modelo, entendida como una entidad abstracta formulada por los científicos, que opera bajo los enunciados de una teoría científica. La relación entre el mundo real y los modelos es la de similaridad, un modelo no es verdadero sino similar al sistema real en algunos aspectos y grados; estos están constituidos por entidades de diversos tipos y de los cuales se realizan una serie de afirmaciones, es decir, en el sistema "pasa lo que las afirmaciones dicen" o, más precisamente, las afirmaciones son verdaderas en dicho sistema. ${ }^{5}$

Desde el campo de la didáctica se ha sustentado que al igual que los científicos los estudiantes generan modelos científicos de "tipo escolar". ${ }^{11}$ Estos modelos son promovidos a partir de una intervención didáctica, que ha sido previamente planeada conceptual y procedimentalmente, la cual se denomina modelización. ${ }^{12} \mathrm{La}$ modelización en el aula propicia en los estudiantes la inmersión en prácticas científicas auténticas, ${ }^{13-15}$ ayudándoles a comprender la ciencia como actividad científica. El operar con modelos o modelizar en el aula promueve en los estudiantes un ejercicio intelectual; esta dinámica lleva al estudiante a comprender y utilizar modelos ya existentes para explicar hechos previamente estudiados por la comunidad científica. ${ }^{11}$ 
En las investigaciones relacionadas con la modelización en el aula se ha determinado que esta actividad se podría categorizar en expresiva, exploratoria, experimental, evaluativa y cíclica, ${ }^{12}$ como se muestra en la Tabla 1.

Tabla 1. Cinco (5) tipos diferentes de modelización

\begin{tabular}{|c|c|c|}
\hline \multirow{6}{*}{ 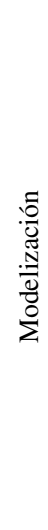 } & Tipo & Característica \\
\hline & Expresiva & $\begin{array}{l}\text { Los estudiantes describen o explican eventos } \\
\text { mediante la formulación de modelos existentes o } \\
\text { nuevos. }\end{array}$ \\
\hline & Exploratoria & $\begin{array}{l}\text { Los estudiantes indagan las características de un } \\
\text { modelo existente participando de este y observando } \\
\text { sus efectos. }\end{array}$ \\
\hline & Experimental & $\begin{array}{l}\text { Los estudiantes formulan hipótesis a partir de } \\
\text { modelos y los ponen a prueba. }\end{array}$ \\
\hline & Evaluativa & $\begin{array}{l}\text { Los estudiantes comparan modelos alternativos que } \\
\text { explican el mismo fenómeno, evalúan sus ventajas } \\
\text { y limitaciones y eligen el más apropiado. }\end{array}$ \\
\hline & Cíclica & $\begin{array}{l}\text { Los estudiantes participan en ciclos completos de } \\
\text { modelización. }\end{array}$ \\
\hline
\end{tabular}

Se ha indicado que a menudo las explicaciones que generan científicos y estudiantes, es sólo la presentación verbal del modelo más adecuado, idea que tendría validez tanto en la actividad científica como en la actividad científica escolar. ${ }^{16}$ En ambas actividades se elaboran modelos, que se construyen y reconstruyen con la pretensión de generar argumentos de los fenómenos que se estudian; al respecto Gilbert et al. proponen una tipología de las explicaciones que generan los científicos, propuesta que permite, también, analizar las explicaciones generadas en el contexto de la actividad científica escolar como se muestra en la Tabla $2 .^{16}$

\section{Marco referencial para el diseño de una propuesta didáctica: generación de modelos explicativos}

El diseño de la propuesta didáctica utilizada en esta investigación se elaboró desde los siguientes referentes teóricos: modelización, andamiaje y enseñar en contexto; lo que permite argumentar el poder conceptual y procedimental del estudio realizado, con el objetivo de caracterizar los modelos explicativos de los futuros médicos en formación (Figura 1).

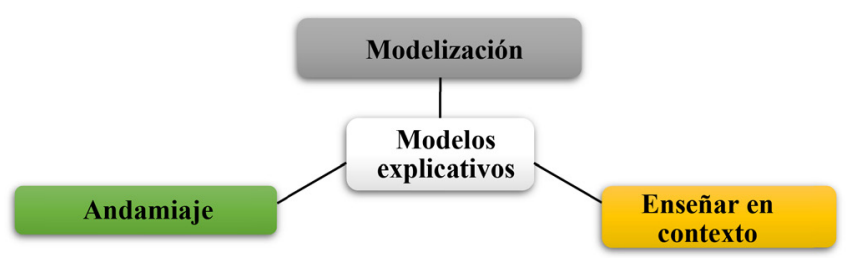

Figura 1. Interacción de componentes teóricos

\section{Enseñar en contexto}

Enseñar química en contexto o contextualizada propende por la formulación de problemas reales y no de simples ejercicios rutinarios, repetitivos y memorísticos, lo que permite recuperar el carácter dinámico y tentativo de la ciencia, mediante actividades específicas de la química en contexto. ${ }^{17}$ Contextualizar la química para enseñarla, significa que el profesor establece una relación entre el conocimiento químico y una situación real desde una intencionalidad didáctica, esta situación le permite al estudiante intervenir en la situación real que demanda por una explicación. ${ }^{18}$

Por lo tanto, una actividad de enseñanza que se diseñe recurriendo a un contexto real deberá cumplir con los objetivos de orientación, motivación, ilustración y aplicación, ${ }^{18}$ estos aseguran la contextualización del modelo científico que se pretende enseñar y la generación de modelos explicativos por parte del estudiantado, garantizando un aprendizaje de calidad, por su factor contextualizante, ${ }^{19}$ ya que la situación de enseñanza contextualizada la asume el estudiante como desafío, insistiendo en su solución, lo cual conlleva a superar obstáculos de aprendizaje y así generar modelos explicativos. ${ }^{20}$

\section{Andamiaje}

En el proceso de aprendizaje se proponen incluir actividades de andamiaje con el objetivo de colaborar en la solución de tareas que no logran resolver por sí mismo al inicio de una temática, actividad que se realiza con la ayuda del profesor. ${ }^{21}$ El propósito de esta colaboración es la de guiar el trabajo de solución de una tarea, utilizando el modelamiento, en donde la mayor parte del trabajo cognitivo es asumido por el docente, mientras el estudiante participa de espectador. A medida que avanza el trabajo en el aula, se le transfiere la responsabilidad de solución de las tareas al estudiante.

Tabla 2. Tipología de modelos explicativos

\begin{tabular}{|c|c|c|}
\hline Modelo explicativo & Pregunta a explicar & Característica \\
\hline Intencional & $\begin{array}{c}\text { ¿Cuál es el propósito por } \\
\text { explicar un fenómeno? }\end{array}$ & $\begin{array}{l}\text { La explicación dada incluye una declaración del propósito que se aborda y otorga una idea de la importancia } \\
\text { del fenómeno que se quiere explicar. En la explicación proporcionada se manifiesta el origen, el alcance y } \\
\text { los límites del fenómeno. En relación con la enseñanza de las ciencias, es una justificación de las acciones } \\
\text { realizadas en la actividad científica escolar por los estudiantes. }\end{array}$ \\
\hline Descriptiva & $\begin{array}{c}\text { ¿Cuáles son las propie- } \\
\text { dades del fenómeno? }\end{array}$ & $\begin{array}{l}\text { Es un resumen de las medidas o características de las partes que componen al fenómeno. Un fenómeno } \\
\text { que aparentemente no cambia se le determina reiteradamente un valor o característica, el cual será tan } \\
\text { cierto como el instrumento lo permita. En relación con la enseñanza de las ciencias, es una aclaración del } \\
\text { significado del fenómeno estudiado por los estudiantes en la actividad científica escolar. }\end{array}$ \\
\hline Interpretativa & $\begin{array}{c}\text { ¿De qué se compone el } \\
\text { fenómeno? }\end{array}$ & $\begin{array}{l}\text { Son declaraciones no solo sobre la naturaleza del fenómeno sino también sobre cómo es su distribución } \\
\text { en el espacio y cómo esa distribución cambia con el tiempo. En otras palabras, los estudiantes recurren a } \\
\text { la teoría para explicar el fenómeno estudiado. }\end{array}$ \\
\hline Causal & $\begin{array}{l}\text { ¿Por qué el fenómeno se } \\
\text { comporta como lo hace? }\end{array}$ & $\begin{array}{l}\text { Es la formulación de un mecanismo explicando el comportamiento observado a través de la operación } \\
\text { de causa y efecto en las entidades de las cuales está compuesto el fenómeno. Es decir, si el estudiante } \\
\text { explica el fenómeno por medio de la causalidad: el fenómeno sucede ya que una causa puede producir } \\
\text { un evento o varios. }\end{array}$ \\
\hline Predictiva & $\begin{array}{l}\text { ¿Cómo se comportará } \\
\text { el fenómeno bajo otras } \\
\text { condiciones específicas? }\end{array}$ & $\begin{array}{l}\text { Una predicción significa anticipar el comportamiento del fenómeno a futuro, partiendo de la idea que será } \\
\text { diferente a uno conocido, pero los modelos construidos permiten tomar decisiones, por ejemplo, generar } \\
\text { una explicación. }\end{array}$ \\
\hline
\end{tabular}


En esta dinámica, el profesor faculta al estudiante a tomar el control sobre el proceso de aprendizaje y asume el papel de espectador.

Los expertos en esta temática han formulado cuatro categorías para comprender el andamiaje, ${ }^{22}$ las cuales se describen a continuación:

1) Metacognitiva: El profesor ha de ayudar a los estudiantes a manejar sus propios pensamientos e ideas durante el trabajo de aprendizaje.

2) Conceptual: El profesor colabora en la construcción de los conceptos acudiendo a los que se aprendieron.

3) Estratégica: El profesor ha de impulsar el análisis, planificación, toma de decisiones y selección de información empleada para conectar lo ya aprendido con la nueva experiencia.

4) Procedimental: El profesor ha de sugerir cómo utilizar fuentes e instrumentos para la solución de la tarea.

\section{Referente químico para la construcción de la propuesta didáctica enfocada en una Situación Problema Contextualizada (SPC)}

Para la SPC se escogió el compuesto etil metil 4-(2,3-diclorofenil)1,4-dihidro -2,6-dimetil-3,5- pyiridinadicarboxilato, también conocido como Felodipino, por ser ampliamente usado en el área de la salud como un bloqueador del canal de dihidroxipyridina de calcio con excelente efecto antihipertensivo. ${ }^{23,24}$ Este medicamento es usado como mezcla racémica, en donde el enantiómero con mayor actividad es el (S)-Felodipino (Figura 2a). ${ }^{25}$ La interacción de este fármaco con la proteína receptora citocromo P450 (PBD ID:2NNJ) (Figura 2b) ya ha sido estudiada y se ha encontrado que es del tipo no covalente, entre las que se destaca la interacción del anillo diclorofenil del Felodipino que se ubica muy cerca del grupo hemo de la proteína, estabilizándose posiblemente por interacciones del tipo $\pi-\pi$. Este mismo grupo presenta otras interacciones importantes con otros residuos de esta proteína, como son la Val366 y Val362, siendo del tipo $\sigma-\pi$. Por lo tanto se dice que las interacciones entre el fármaco y la proteína son predominantemente hidrofóbicas. ${ }^{26}$ Sin embargo, se presentan interacciones del tipo dipolo - dipolo, destacándose la formación de enlaces de hidrogeno con los residuos de los aminoácidos SER100 y SER103 (Figura 2c). Otro aspecto destacado para entender la relación entre el Felodipino y la proteína receptora son las propiedades termodinámicas, tales como la entalpía $(\Delta \mathrm{H})$, la entropía $(\Delta \mathrm{S})$ y energía libre de $\operatorname{Gibbs}(\Delta \mathrm{G})$. Es bien conocido que para lograr una interacción energéticamente favorable entre dos sustancias es suficiente con tener un valor negativo de $\Delta \mathrm{G}$, sin embargo, físicamente este se deriva de los valores de $\Delta \mathrm{H}$ y $\Delta \mathrm{S}$, lo que implica analizar a fondo estos conceptos. La entalpía es una propiedad termodinámica que refiere al intercambio de energía entre un sistema y sus alrededores, pero también es muy común relacionarlo con las energía involucradas en la formación de enlaces y las interacciones a nivel molecular, indicándonos que cuando tenemos un valor negativo estamos frente a un proceso exotérmico, pero aún más importante, estamos hablando de un proceso en el que los complejos o productos formados presentan valores energéticos menores a los de sus precursores, es decir son más estables y por tanto suele ser un proceso favorable. Pero el factor entálpico no siempre es suficiente para decidir si un proceso en general es espontaneo, para esto también se necesita el factor entrópico, dado que esta información es relevante para comprender las distintas configuraciones que adoptan las moléculas y átomos antes y después de un proceso dado, siendo natural un aumento en este valor, por lo que valores altos de entropía se asocian a procesos espontáneos. Ahora, estos dos factores combinados generan información suficiente para comprender el $\Delta \mathrm{G}$, es decir, información que permite predecir cuan favorable es un proceso. ${ }^{27}$

El anterior referente teórico orientó el tipo de modelización que se pretendió promover durante la investigación y el análisis de los modelos explicativos que formulan los estudiantes del programa medicina de primer año de la Universidad del Norte de Barranquilla - Colombia, al solucionar reiteradamente una SPC, mediante el análisis de las interacciones químicas que se generan entre el Felodipino y el citocromo P450, interacciones que se comprenden desde conceptos generales de la química. El grupo de investigación diseñó la propuesta didáctica enfocada a la formación inicial de médicos con el propósito de promover la formulación de modelos explicativos en el estudiantado de este programa académico. Los interrogantes que orientaron la investigación fueron: ¿Qué tipos de modelos explicativos construyen los estudiantes del programa de medicina al resolver la SPC? y ¿Cuál es el modelo explicativo más recurrente en los estudiantes al solucionar la SPC? a)

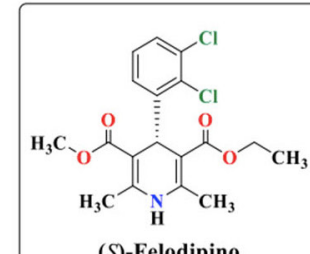

(S)-Felodipino

$$
(R) \text {-Felodipino }
$$

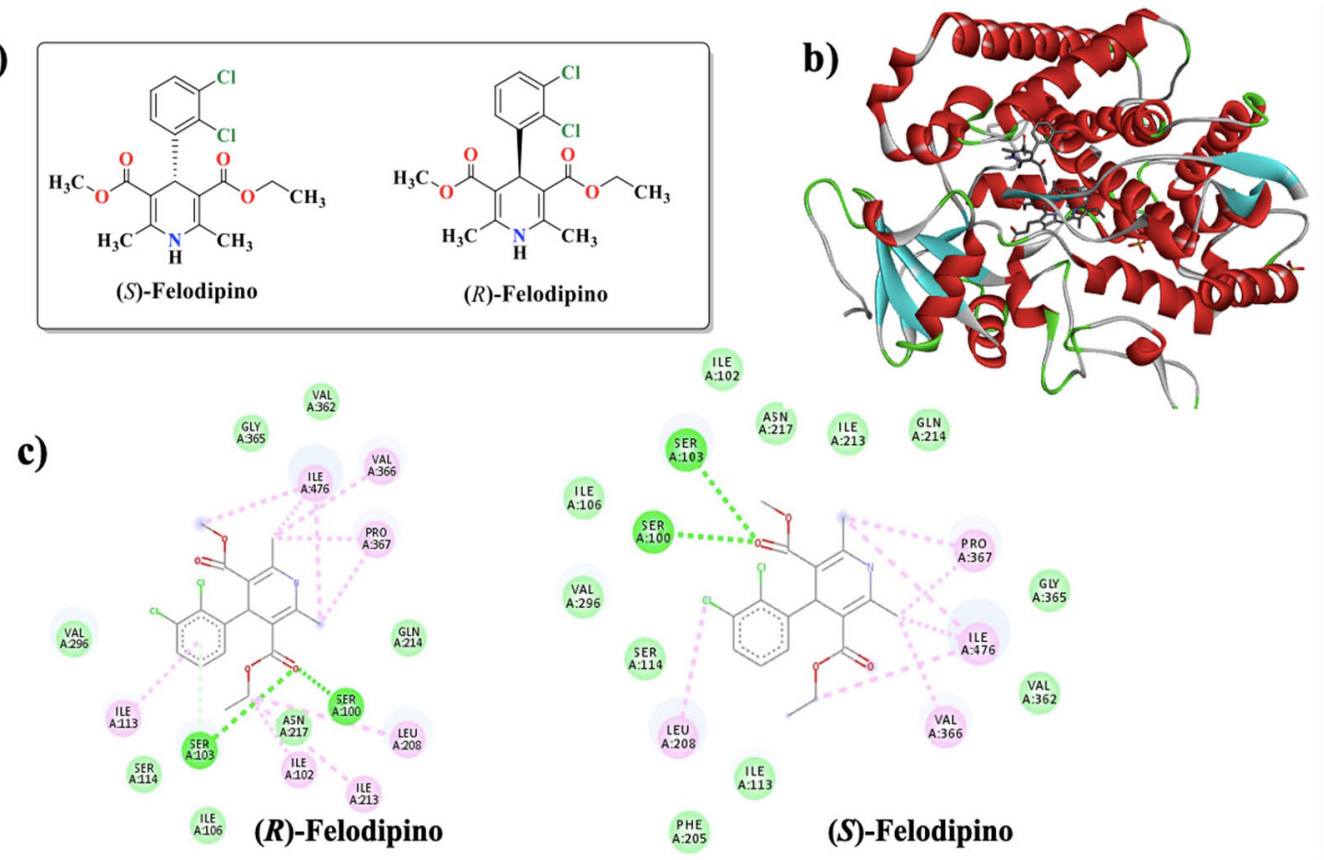

Figura 2. a) Estructuras del Felodipino, b) proteína PBD ID:2NNJ interaccionando con la molécula del Felodipino y c) principales interacciones entre los residuos de aminoácidos de la proteína PBD ID:2NNJ y el Felodipino 


\section{METODOLOGÍA}

La investigación se enmarca en la metodología cualitativa, lo cual permite estudiar en profundidad un fenómeno en un contexto específico. ${ }^{28}$ Para la determinación de los modelos explicativos se recurrió al método de triangulación, técnica que permite la determinación de patrones de convergencia para poder desarrollar o corroborar una interpretación global del fenómeno objeto de la investigación, específicamente se implementó la de investigadores, la cual es llevada a cabo por diferentes personas, actividad que permite reducir los sesgos en la investigación por un único investigador en la recolección y análisis de datos y consistencia en los hallazgos. ${ }^{29}$.

En resumen, esta investigación se desarrolló en diferentes etapas, la primera consistió en la construcción de la SPC, seguido de su implementación con los estudiantes de medicina en diferentes momentos del semestre. Finalmente, los datos obtenidos fueron analizados por el método de triangulación de investigadores. A continuación, se describe cada una de estas etapas.

\section{Diseño de la SPC}

Atendiendo a los problemas de una enseñanza de la química descontextualizada, se diseñó la propuesta didáctica centrada en la modelización de tipo expresiva (Tabla 1), relacionada con la interacción entre el felodipino y la proteína PBD ID:2NNJ, vinculando los conceptos que previamente el grupo de investigación seleccionó como claves para un curso de química para la formación de médicos. Los conceptos elegidos fueron: Interacciones intermoleculares, energía libre de Gibbs e isomería (Figura 3). El trabajo de discusión se centró en el análisis del contenido químico, se examinó con detalle las temáticas propuestas para el curso de química para el programa de medicina y se fue depurando con el propósito de seleccionar el de mayor argumentación a la SPC. Hecha la selección de las temáticas que a juicio del grupo de investigación presentaban mayor vinculación conceptual con la SPC se procedió al ajuste de esta, en términos de la selección del contenido específico de cada temática elegida, tal acople se realizó desde dos intencionalidades una química y otra didáctica. Desde el aprendizaje de estos conceptos los estudiantes construyeron modelos explicativos como respuestas a la SPC. La especificidad de cada uno de los investigadores permitió la construcción de la situación de enseñanza contextualizada con una impronta desde el conocimiento químico y desde la didáctica de la química (Material Suplementario).

\section{Implementación de SPC}

En la Figura 4, se muestra el proceso de implementación de la SPC, desde una enseñanza contextualizada basada en la modelización y orientada pragmáticamente por la idea de andamiaje, en el que se modeló la interacción del ibuprofeno como un buen ejemplar de comprensión por parte del profesor.

La SPC se implementó a 81 estudiantes de primer año del programa de medicina de la Universidad del Norte. Se informó que harían parte de una investigación didáctica que duraría todo el semestre de estudio y que estaría relacionada con su formación académica. Adicionalmente, diligenciaron un formato de consentimiento en el cual aceptaban participar en la investigación.

La implementación de las SPC se hizo en cuatro momentos denominados M1, M2, M3 y M4 (Figura 4). La M1 se efectuó el primer día de clase, determinando los modelos explicativos previos de los estudiantes sin realizar ninguna intervención didáctica con el grupo de estudiantes. Posteriormente, se llevó a cabo el desarrollo de la temática enlace químico y se procedió por parte del profesor a desarrollar la actividad de andamiaje modelando las interacciones entre el fármaco Ibuprofeno con la Cycloxigenasa-2. El objetivo de este ejemplo fue orientar la comprensión de la temática enlace químico vista con una situación contextual al campo de formación de los estudiantes, una vez finalizado el tema se implementó la SPC (M2). Luego, se llevó a cabo el desarrollo de la temática termoquímica y se realizó el modelado con Ibuprofeno haciendo énfasis en las propiedades termodinámicas, tales como la entalpía $(\Delta \mathrm{H})$, la entropía

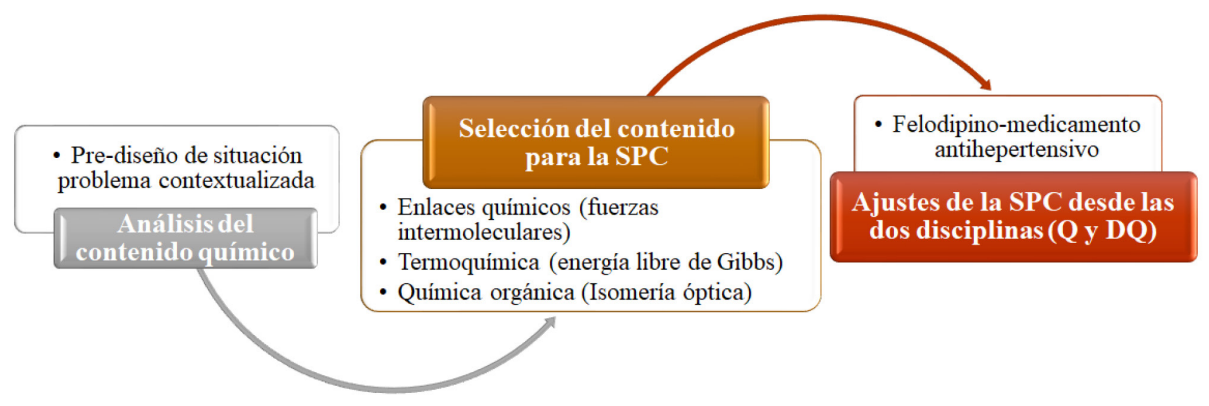

Figura 3. Proceso de diseño de la SPC

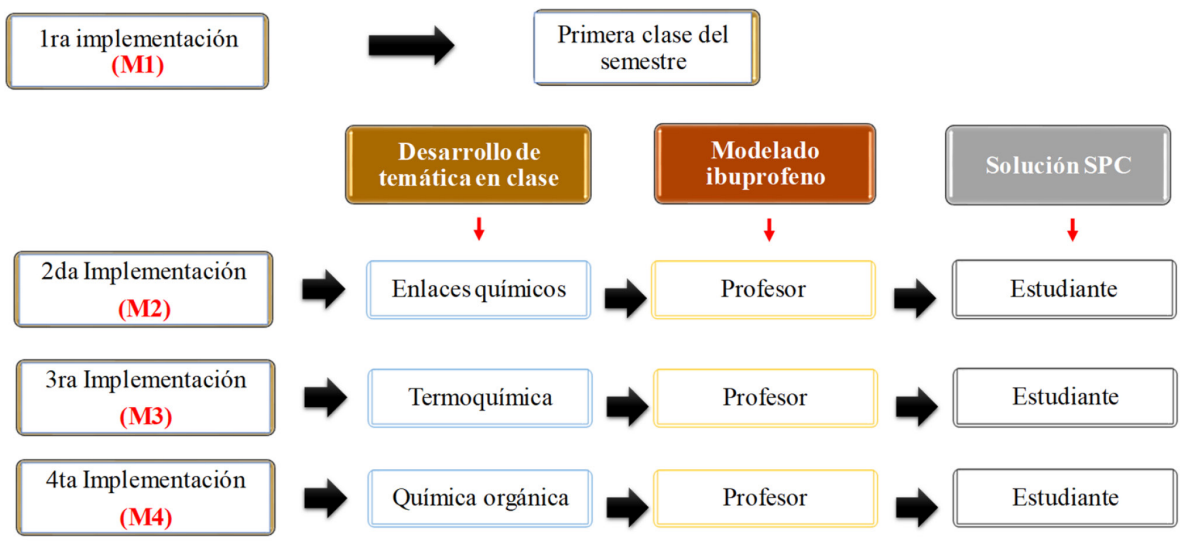

Figura 4. Implementación de la SPC 
$(\Delta S)$ y energía libre de Gibbs $(\Delta \mathrm{G})$. Una vez terminada la explicación, se presentó nuevamente la SPC para que generen sus modelos explicativos (M3); para el último momento (M4) se desarrolló la temática química orgánica, y se presentó el modelado con Ibuprofeno acentuando en la temática de isomería óptica. Finalmente, se aplicó la SPC para que generaran sus modelos explicativos.

Para los momentos 2, 3 y 4 los estudiantes tenían la oportunidad de consultar sus respuestas anteriores, con el propósito de que evaluaran sus explicaciones e hicieran los ajustes necesarios al nuevo modelo explicativo que construirían. Para el desarrollo de la SPC en cada momento los estudiantes tenían un tiempo estimado de media hora de clase. Las respuestas a la SPC de los estudiantes eran escritas y entregadas al profesor, para que así se iniciara el proceso de análisis por parte del equipo de investigación

\section{Metodología para analizar los datos obtenidos de las SPC}

El proceso utilizado para el análisis de los modelos explicativos, iniciaba después que los estudiantes generaban sus modelos en cada momento de la implementación. Cada miembro del grupo de investigación analizó las respuestas elaboradas por los estudiantes con el propósito de identificar con cual modelo explicativo presentaban mayor afinidad (ver Tabla 2); el proceso se realizó en los 4 momentos de la implementación de la propuesta didáctica. Posteriormente se procedió a triangular los análisis individuales con el propósito de determinar la relación de la tipología de modelo explicativo (Figura 5).

\section{RESULTADOS Y DISCUSIÓN}

La organización y análisis de los datos se realizó desde lo cualitativo, divididos en dos etapas. En la primera etapa se efectuó la organización de los datos, identificando los que cumplen con la condición de ser un modelo explicativo y posteriormente su vinculación semánticamente con algún tipo de modelo explicativo de la Tabla 2, los resultados se muestran en la Figura 6. Se aclara que ninguno de los modelos explicativos propuestos por los estudiantes en los cuatro momentos cumple con la descripción de un modelo explicativo intencional como se define en la Tabla 2. Es importante resaltar que se analizó la generación de modelos explicativos de los estudiantes, por lo que la investigación no pretende generalizar resultados y conclusiones a una población más amplia. Sin embargo, los resultados pueden compararse con los de otros trabajos en los que estén implicados participantes de otros contextos, y al encontrar patrones comunes permitiría un cierto grado de generalización.

A continuación, se presentan ejemplares de modelos explicativos construidos por los estudiantes, específicamente de tipo Interpretativo y Descriptivo, siendo los de mayor recurrencia en la investigación (Tabla 3 y 4 ).

La organización de los datos (respuestas de los estudiantes) y el posterior análisis permitió determinar que en el primer momento

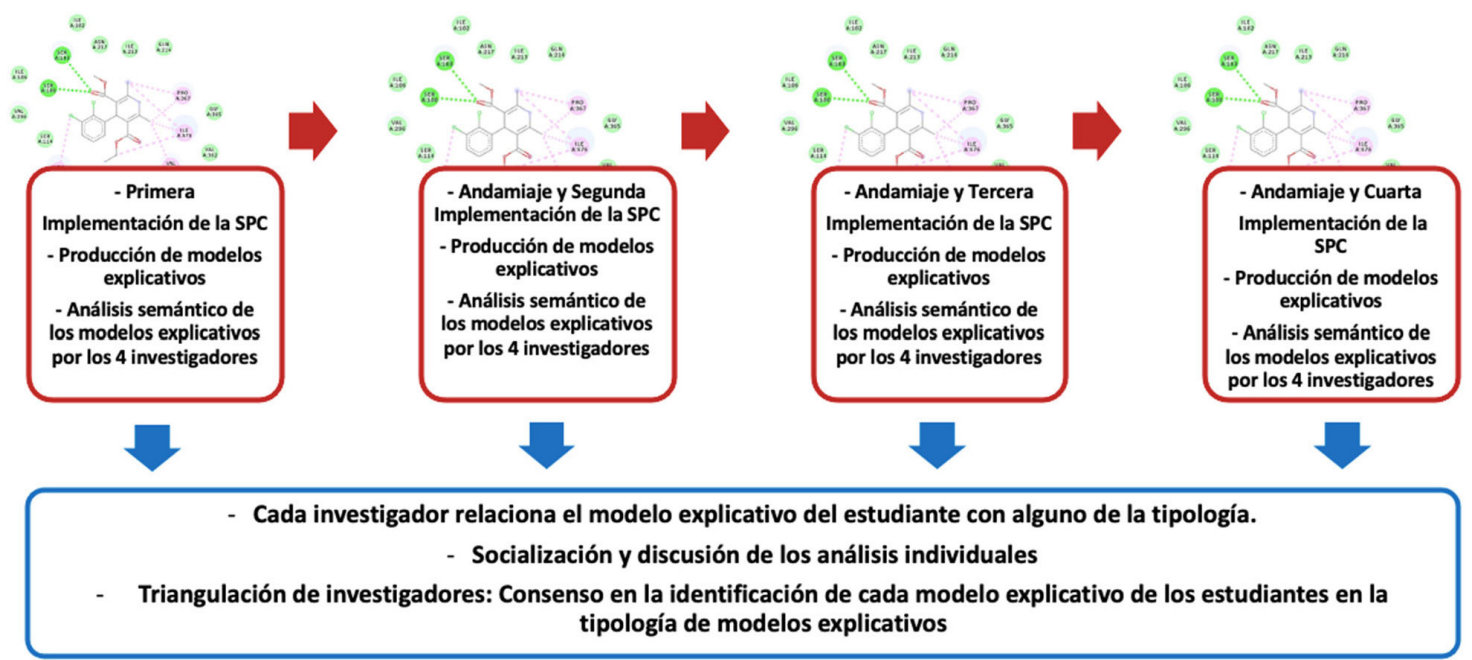

Figura 5. Descripción del proceso de análisis de los datos

Tabla 3. Ejemplares de Modelos Explicativos Interpretativos (MEI)

\begin{tabular}{cl}
\hline Momento & \multicolumn{1}{c}{ Ejemplos de respuestas construidas por los estudiantes } \\
\hline 2 & $\begin{array}{l}\text { El Felodipino interactúa con la enzima 2NNJ y crea puentes de hidrógeno lo cual no permite que se den } \\
\text { las uniones normales. }\end{array}$ \\
\hline $\begin{array}{l}\text { La interacción de fármacos con enzimas o proteínas tiene relación directa con las entalpias y su impacto } \\
\text { en la energía libre de Gibbs; para que un fármaco sea efectivo, siempre deberá existir una energía negativa, } \\
\text { que facilite las condiciones de interacción, además deberá haber una disminución de la entropía, pues el } \\
\text { desorden cuando dos sustancias se unen tiende a disminuir. Impacto exitoso de las sustancias es igual a } \\
\text { una energía libre Gibbs negativa. }\end{array}$
\end{tabular}

El Felodipino ingresa al organismo y a la célula haciendo un complejo enzima-sustrato con la enzima 2NNJ, para que se de esa interacción se requiere de fuerzas intermoleculares y electroestáticas. Los puentes de hidrogeno son la clave para la interacción de estos dos componentes ya que el grupo carboxilo del aminoácido con el grupo $\mathrm{OH}$ presente en varios radicales, pudiendo interactuar el hidrogeno $(\mathrm{H})$ del Felodipino con el oxígeno del aminoácido o viceversa. Cabe resaltar que esta interacción hace que el desorden disminuya y por ende aumente la energía libre de Gibbs, este aumento depende en gran medida de cual configuración (cual isómero) interactúe con la enzima, ya que como mencionamos la interacción química de cada uno será diferente y tendrá una estabilidad diferente, siendo el que libera menor energía de Gibbs (A) el que tiene más estabilidad viene siendo este un proceso más espontaneo.

Vinculación con el Modelo Explicativo Interpretativo 
Tabla 4. Ejemplares de Modelos Explicativos Descriptivos (MED)

\begin{tabular}{|c|c|c|}
\hline Momento & Ejemplos de respuestas construidas por los estudiantes & \\
\hline 2 & El Felodipino crea un puente de hidrogeno y un enlace dipolo-dipolo en una misma zona. & \\
\hline 3 & $\begin{array}{l}\text { Todo esto genera que se forme el complejo Felo-enzima, disminuyendo el } \Delta \mathrm{S} \text {, lo que libera energía porque } \\
\text { es una reacción de formación } \Delta \mathrm{H}<0 \text { : como resultado la } \Delta \mathrm{G} \text { es negativo por lo que es una reacción espon- } \\
\text { tánea } \Delta \mathrm{G}<0 \text {. }\end{array}$ & \\
\hline 4 & $\begin{array}{l}\text { El fármaco y el complejo enzimático se unen mediante fuerzas intermoleculares distintas, dependiendo de } \\
\text { los grupos funcionales -ubicados en sitio activo de la enzima- con los que actúa el Felodipino. Por ejemplo, } \\
\text { si encontramos entre grupos funcionales un } \mathrm{OH} \text {, claramente al formarse liberan energía, haciendo que la } \\
\text { entalpía sea negativa (cabe decir, la interacción del medicamento con la enzima es dominado por el calor } \\
\text { generado), es decir, es una reacción exotérmica. La energía libre de Gibbs, depende por su parte de la en- } \\
\text { talpía, siendo así negativa (menor que 0) y definiendo la interacción del complejo y fármaco como reacción } \\
\text { espontánea. La entropía disminuye en la reacción, por que disminuye el desorden del sistema. Analizando las } \\
\text { estructuras de los isómeros de Felodipino, podemos decir que el isómero A es más activo que el isómero B. } \\
\text { (Isómero A=R; Isomero B=S). Determinamos esto por la energía de Gibbs de tales isómeros. }\end{array}$ & $\begin{array}{l}\text { Vinculación con el Modelo } \\
\text { Explicativo Descriptivo }\end{array}$ \\
\hline
\end{tabular}

(M1) 79 de 81 respuestas se catalogaron como ningún modelo explicativo (NME), es decir, estos datos no están relacionados con ninguna de las categorías de modelo explicativo que se propuso para la investigación. En el segundo momento (M2) de la implementación el número de NME disminuye considerablemente de 79 a 31 de los 81 datos. Se determinó que 40 respuestas cumplen con características de MED, 8 con MEI, 1 con MEP y 1 con MEC. Para el tercer momento (M3) de implementación se determinaron 30 NME de los 81 datos, lo que nos muestra una tendencia a la disminución de los NME y al aumento de modelos explicativos, 27 MED, 23 MEI y solo 1 MEC. En lo que respecta al último momento (M4), continúa la disminución del número de NME y el aumento en MEI (32), MED (31), MEP (2) y MEC (2) como se muestran en la Tabla 3 y en el Figura 6.

En un segundo nivel de análisis, se realizó una aproximación heurística para caracterizar los modelos explicativos desde el conocimiento químico (análisis cualitativo), a partir de la organización

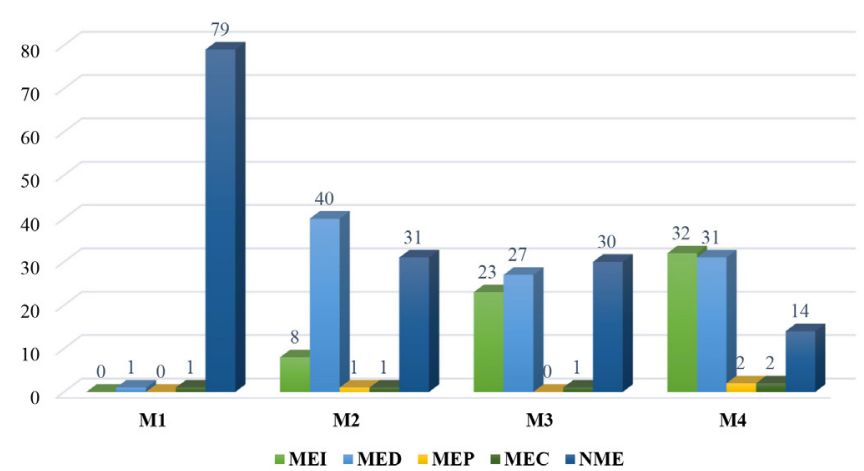

Figura 6. Modelos explicativos de los estudiantes identificados en cada momento de la implementación

de los datos en la etapa 1, determinando recurrencias en cada grupo de modelos explicativos. En las Tablas 5, 6, 7 y 8 se presentan algunas

Tabla 5. Ejemplares de Modelos Explicativos Interpretativos y su interpretación desde la química

\begin{tabular}{|c|c|c|}
\hline \multirow{2}{*}{ Momento } & \multicolumn{2}{|c|}{ Modelo Explicativo Interpretativo (MEI) } \\
\hline & Ejemplos de respuestas construidas por los estudiantes & Análisis desde el contenido químico de los MEI de los estudiantes \\
\hline 2 & $\begin{array}{l}\text { Existen fuerzas intermoleculares que hacen posible la interacción } \\
\text { entre ellos. Pueden existir enlaces por puente de hidrógenos pues con } \\
\text { aminoácidos tienen el grupo amino que puede enlazarse por este medio } \\
\text { y también ambos son moléculas polares por lo que podrían interaccio- } \\
\text { nar por enlaces dipolo-dipolo, son polares por la presencia de átomos } \\
\text { altamente electronegativos. }\end{array}$ & $\begin{array}{l}\text { Estos modelos recurren a la noción de enlace para resaltar la existencia } \\
\text { de distintas fuerzas intermoleculares en las dos estructuras químicas del } \\
\text { felodipino haciendo énfasis en la formación de fuerzas dipolo-dipolo } \\
\text { y puente de hidrógeno. }\end{array}$ \\
\hline
\end{tabular}
altamente electronegativos

El A tiene una mayor interacción molecular, la entropía disminuye, ya que interacciona con dos aminoácidos, este compuesto, tiene una entropía (al interaccionar con la enzima). - $\Delta$ S. Por lo tanto, su $\Delta \mathrm{G}$ será negativo. El B tiene una interacción molecular con la enzima respecto al $\mathrm{A}$, pero al ser solo una su $\Delta \mathrm{G}$ igual será negativo, pero mayor al $\mathrm{A}$. Por lo tanto, el A tendrá una mayor eficacia al inhibir la cadena de transferencia electrónica en la enzima porque su $\Delta \mathrm{G}$ será más negativo que el $\Delta \mathrm{G}$ del $\mathrm{B}$.

El Felodipino interactúa químicamente adhiriéndose a la cadena de aminoácidos que se encuentra en el sitio activo de la enzima $2 \mathrm{NNJ}$, la cual cataboliza la dilatación de arterias periféricas y logra inhibir la cadena de transferencia electrónica. Pueden existir enlaces como puentes de hidrógeno entre ambas cosas. El hecho de que existan enantiómeros distintos para el fármaco quiere decir que no se unirán igual a la enzima, ya que tendría secuencias diferentes, es por esto que tendrían energía libre de Gibbs diferentes. La unión entre el Felodipino y una enzima está definida por su entalpía $\Delta H$, al unirse los dos está estaría disminuyendo por los que se energía libre de Gibbs sería menor a 0 , naciendo la interacción espontanea. También, la entropía estaría disminuyendo ya que las moléculas sueltas se organizarían el

Estos modelos recurren a nociones de termoquímica y de fuerza intermolecular para establecer distinción en las estructuras del felodipino y aclarar que cada estructura interactúa diferente, interpretando valores de entalpía, entropía y energía libre de Gibbs. unirse al fármaco.

Estos modelos refieren acuden a nociones de fuerzas intermoleculares, termoquímica e isomería para explicar la interacción de los dos isómeros del felodipino vinculando los valores de energía libre de Gibbs e interpretando la entalpía y la entropía de cada estructura del felodipino. 
Tabla 6. Ejemplares de Modelos Explicativos Descriptivos y su interpretación desde la química

\begin{tabular}{|c|c|c|}
\hline \multirow{2}{*}{ Momento } & \multicolumn{2}{|c|}{ Modelo Explicativo Descriptivo (MED) } \\
\hline & Ejemplos de respuestas construidas por los estudiantes & Análisis desde el contenido químico de los MED de los estudiantes \\
\hline 2 & $\begin{array}{l}\text { Compuesto A: ingresa la proteína e interactúa con la treonina y con la } \\
\text { asparagina. Formando puentes de hidrógeno e interacciones dipolares. } \\
\text { Compuesto B. Ingresa la proteína e interactúa fuertemente con la } \\
\text { treonina. Forma también puentes de hidrógeno e interacciones } \pi-\pi \text {. }\end{array}$ & $\begin{array}{l}\text { Estos modelos aluden a la noción de fuerzas intermoleculares de tipo } \\
\text { puente de hidrógeno y/o dipolo-dipolo para detallar la existencia de } \\
\text { estas fuerzas entre los isómeros del felodipino y la proteína. }\end{array}$ \\
\hline 3 & $\begin{array}{l}\text { Todo esto genera que se forme el complejo felo-enzima, disminuy- } \\
\text { endo el } \Delta \mathrm{S} \text {, lo que libera energía porque es una reacción de formación } \\
\Delta \mathrm{H}<0 \text { : como resultado la } \Delta \mathrm{G} \text { es negativo por lo que es una reacción } \\
\text { espontánea } \Delta \mathrm{G}<0 \text {. }\end{array}$ & $\begin{array}{l}\text { Estos modelos recurren a nociones de energía libre de Gibbs, entropía } \\
\text { y/o entalpía (termoquímica) para relatar la reacción entre el felodipino } \\
\text { y la enzima. }\end{array}$ \\
\hline
\end{tabular}

El fármaco y el complejo enzimático se unen mediante fuerzas intermoleculares distintas, dependiendo de los grupos funcionales -ubicados en sitio activo de la enzima- con los que actúa el felodipino.

Por ejemplo, si encontramos entre grupos funcionales un $\mathrm{OH}$, claramente se forma liberan energía, haciendo que la entalpía sea negativa (cabe decir, la interacción del medicamento con la enzima es dominado por el calor generado), es decir, es una reacción exotérmica. La energía 4 libre de Gibbs, depende por su parte de la entalpía, siendo así negativa (menor que 0) y definiendo la interacción del complejo y fármaco como reacción espontánea.

La entropía disminuye en la reacción, porque disminuye el desorden del sistema. Analizando las estructuras de los isómeros de felodipino, podemos decir que el isómero $\mathrm{A}$ es más activo que el isómero $\mathrm{B}$. Isómero $\mathrm{A}=\mathrm{R}$; Isómero $\mathrm{B}=\mathrm{S}$. Determinamos esto por la energía de Gibbs de tales isómeros.

Tabla 7. Ejemplares de Modelos Explicativos Causales y su interpretación desde la química

\begin{tabular}{|c|c|c|}
\hline \multirow{2}{*}{ Momento } & \multicolumn{2}{|c|}{ Modelo Explicativo Causal (MEC) } \\
\hline & Ejemplos de respuestas construidas por los estudiantes & Análisis desde el contenido químico de los MEC de los estudiantes \\
\hline 4 & $\begin{array}{l}\text { El felodipino A es } \mathrm{S} \text {, debido a esto es correcto decir que tiene mayor } \\
\text { fuerza de interacción por eso su } \Delta \mathrm{G} \text { es menor. Mientras que el felodipino } \\
\mathrm{B} \text { es } \mathrm{R} \text { porque no tiene mayor fuerza de interacción que el felodipino } \\
\mathrm{A} \text {, por eso es que su } \Delta \mathrm{G} \text { es mayor que el del felodipio A }\end{array}$ & $\begin{array}{l}\text { Estos modelos recurren a nociones de fuerzas intermoleculares, termo- } \\
\text { química e isomería para explicar cómo se origina la interacción entre las } \\
\text { estructuras del felodipino y la proteína, apoyándose en la comprensión } \\
\text { de los conceptos de energía libre de Gibbs, entropía y/o entalpía. }\end{array}$ \\
\hline
\end{tabular}

Tabla 8. Ejemplares de Modelos Explicativos Predictivos y su interpretación desde la química

\begin{tabular}{|c|c|c|}
\hline \multirow{2}{*}{ Momento } & \multicolumn{2}{|c|}{ Modelo Explicativo Predictivo (MEP) } \\
\hline & Ejemplos de respuestas construidas por los estudiantes & Análisis desde el contenido químico de los MEP de los estudiantes \\
\hline 4 & $\begin{array}{l}\text { Existen fuerzas intermoleculares que hacen posible la interacción } \\
\text { entre ellas. Pueden existir enlaces por puentes de hidrógeno pues } \\
\text { los aminoácidos tienen el grupo amino que puede enlazarse por este } \\
\text { medio. Otro aspecto es que ambas son moléculas polares por lo que } \\
\text { podrían enlazarse dipolo-dipolo y son polares por la presencia de } \\
\text { átomos altamente electronegativos. Así mismo se presenta un cambio } \\
\text { en la energía libre de Gibbs, pues sus componentes, en este caso la } \\
\text { entalpía y entropía se hace más negativa y se reduce respectivamente } \\
\text { al momento de la unión. Esto debido a que la unión libera energía y el } \\
\text { fármaco pasa a un estado con menos desorden. }\end{array}$ & $\begin{array}{l}\text { Estos modelos aluden a nociones de termoquímica, isomería y noción } \\
\text { de fuerza intermolecular para predecir que uno de los compuestos del } \\
\text { felodipino pueda tener mayor número de interacciones al analizar la } \\
\text { presencia de puentes de hidrógeno entre aminoácidos y el felodipino, } \\
\text { los valores de entalpía, entropía y energía libre de Gibbs }\end{array}$ \\
\hline
\end{tabular}

de las respuestas y se especifica el momento de estas, además se muestran los análisis de los modelos explicativos desde lo químico.

La Figura 6 muestra en cierta medida la eficacia de la propuesta didáctica formulada desde la idea de enseñanza en contexto basada en la modelización. Gradualmente aumenta la formulación de modelos explicativos en los estudiantes una vez se inicia con la intervención didáctica, desde el momento 1 (M1) al 4 (M4) de la implementación didáctica (Figura 4). Se resalta que las respuestas generadas en M1 no cumplen con los criterios propuestos en la investigación (Tabla 2). Tan solo 2 respuestas de 81 cumplen con las características de modelo explicativo, uno concordando con el tipo predictivo y el otro con el causal. Dado que en el M1 no hubo ninguna interacción entre el grupo de estudiantes y el docente, estos tuvieron que recurrir a sus conocimientos previos para resolver la SPC y los cuales han sido elaborados por su tránsito en la educación primaria y secundaria, siendo semánticamente pobres para catalogarlos en algunos de las tipologías de modelo explicativo.

Se considera que los resultados obtenidos en los momentos 2, 3 y 4, se generan a causa de dos factores, uno a la coherencia teóricodidáctica desde la cual se propuso la SPC, soportada desde los pilares de la modelización, la enseñanza en contexto y el andamiaje. Tal congruencia se logra por la reflexión desde el conocimiento químico y didáctico del grupo de investigación que realiza su actividad científica en uno u otro campo de conocimiento, situación que permitió el diseño de la SPC para una enseñanza en contexto basada en la modelización. En relación con el andamiaje, se considera pieza importante en la intervención didáctica, ya que, al finalizar el desarrollo de cada temática, el docente recurrió al caso del ibuprofeno como un buen 
"ejemplar" para enseñar la actividad de un fármaco, analizado desde los modelos científicos de la química general. El segundo factor fue la implementación de la misma SPC durante toda la intervención, permitiéndole al estudiantado evaluar y re-evaluar sus modelos explicativos, aspecto que promovió el robustecimiento de los modelos en los estudiantes, afirmación que se sostiene en el análisis de las respuestas del momento 4 (ver Tablas 5, 6, 7 y 8), 14 respuestas de los estudiantes no concuerdan con los parámetros de algún modelo explicativo, mientras que 67 respuestas cumplen con las condiciones necesarias para categorizarlos.

Finalmente, se hicieron análisis secundarios permitiendo inferir que los modelos explicativos de los estudiantes en los momentos 2 , 3 y 4 los elaboran con contenido químico, permitiendo colegir que no solo construyen modelos explicativos coherentes, sino que están cargados de contenido, principalmente los elaborados en el momento 4. Es en este momento en el que la mayoría de los estudiantes formulan modelos explicativos vinculando contenidos de enlace químico, termoquímica e isomería (Ver Tabla 9 y 10).

Tabla 9. Resaltando el contenido químico de Modelos Explicativos Interpretativos

\section{Algunos modelos Recurre a las nociones de: \\ Fuerzas intermoleculares, termoquímica e isomería para explicar la interacción de las dos estructuras del felodipino desde los valores de energía libre de Gibbs vinculados a los isómeros $\mathrm{R}$ y $\mathrm{S}$ del felodipino. \\ Fuerzas intermoleculares, termoquímica e isomería para explicar la interacción de las dos estructuras del felodipino desde los valores de energía libre de Gibbs vinculados a los isómeros del felodipino.}

Termoquímica y de fuerza intermolecular para establecer distinción en las estructuras del felodipino y aclarar que cada estructura interactúa diferente, interpretando valores de entropía.

Termoquímica para establecer distinción en las estructuras del felodipino y aclarar que cada estructura interactúa diferente, interpretando valores de energía libre de Gibbs.

Fuerza intermolecular y de termoquímica para explicar la interacción del felodipino interpretando valores de entalpía y entropía.

Fuerzas intermoleculares, termoquímica e isomería para explicar la interacción de las dos estructuras del felodipino desde los valores de entalpía, entropía y energía libre de Gibbs vinculados a los isómeros del felodipino.

Fuerzas intermoleculares y termoquímica para explicar la interacción del felodipino desde los valores de entalpía, entropía y energía libre de Gibbs.

Fuerzas intermoleculares y termoquímica para explicar la interacción de las estructuras del felodipino desde los valores de energía libre de Gibbs.

Enlace para resaltar la existencia de distintas fuerzas intermoleculares en el felodipino haciendo énfasis en la formación de puentes de hidrógeno.

Fuerzas intermoleculares, termoquímica e isomería para explicar la interacción del felodipino desde el concepto de energía libre de Gibbs vinculado al concepto de isómero.

Fuerzas intermoleculares e isomería para explicar la interacción del felodipino vinculado el concepto de isómero.

Termoquímica e isomería para explicar la interacción del felodipino vinculado los conceptos de entropía, entalpía e isomería.

Fuerzas intermoleculares, termoquímica e isomería para explicar la interacción de las dos estructuras del felodipino desde los valores de entalpía y entropía vinculados a los isómeros del felodipino.

Fuerzas intermoleculares, termoquímica e isomería para explicar la interacción del felodipino desde el concepto de entropía vinculado al concepto de isómero.
Tabla 10. Resaltando el contenido químico de Modelos Explicativos Descriptivos

\begin{tabular}{ll}
\hline Algunos modelos Recurre a las nociones de: \\
$\begin{array}{l}\text { Energía libre de Gibbs, entropía y/o entalpía (termoquímica) y de } \\
\text { isomería para describir la reacción entre las estructuras del felodipino } \\
\text { y la proteína. }\end{array}$ \\
$\begin{array}{l}\text { Interacción intermolecular, energía libre de Gibbs, entropía y/o en- } \\
\text { talpía (termoquímica) y de isomería para describir la reacción entre }\end{array}$ \\
las estructuras del felodipino y la proteína. \\
\hline
\end{tabular}

\section{CONCLUSIONES}

La estrategia de enseñanza implementada fue exitosa, debido a que aumento gradualmente la formulación de los modelos explicativos una vez iniciada la intervención didáctica, inicialmente solo el $2.46 \%$ de estudiantes formularon modelos explicativos y al finalizar la implementación el $82.7 \%$ de estudiantes elaboran modelos explicativos robustos con fundamentos de la química, lo que permite inferir que enseñar en contexto es importante para la formación de profesionales en área de la salud.

Los modelos explicativos que generan los estudiantes de un fenómeno han de estar cargados de teoría, puntualmente para esta investigación en la que se diseñó e implemento una SPC desde la idea de modelización de tipo expresiva en la que los estudiantes describen o explican eventos mediante la formulación de modelos existentes o nuevos, se determinó que la recurrencia de los estudiantes por formular modelos explicativos es de tipo: Descriptivo e Interpretativo; identificando que los modelos de los estudiantes progresivamente aumentaban en su carga teórica desde el conocimiento químico, situación que se evidencia en los análisis primarios y sobre todo en los secundarios.

La propuesta didáctica diseñada desde la perspectiva de enseñanza en contexto basada en la modelización y apalancada por andamiaje resulto pertinente para la transformación de modelos explicativos en médicos de primer año en formación, es así como se pasó de 79 respuestas de los estudiantes no categorizables como modelos explicativos a 67 respuestas vinculadas con algún tipo de modelo explicativo, mayormente de tipo Descriptivo o Interpretativo. Se considera que la propuesta de intervención diseñada para la formación de médicos es potente tanto en lo teórico como en lo metodológico, ya que se aleja de la postura tradicional de enseñanza, en la que el docente se concentra en transmitir contenidos de las ciencias sin ninguna vinculación con un contexto definido. La propuesta no solo estuvo relacionada con la enseñanza en contexto, además promovió la construcción y reconstrucción de modelos explicativos a partir del abordaje de los contenidos de la química, del estudio de un buen "ejemplar" y de la solución de una misma SPC a lo largo de un trabajo didáctico, pensado especialmente para la formación de un profesional de la salud, aspectos que permitieron en cierta medida el éxito de la investigación.

Se confirma que estructurar la actividad química escolar entorno a modelos científicos de la ciencia química, permite generar en el aula un saber químico que les posibilita a los estudiantes comprender 
el funcionamiento del mundo y más cuando se enseña en contextobasada en la modelización, permitiéndoles construir modelos explicativos similares a los que producen los científicos en su actividad, con el objetivo de representar, argumentar e intervenir en su ámbito de formación profesional.

\section{MATERIAL SUPLEMENTARIO}

La situación problema contextualizada (SPC) está disponible en http://quimicanova.sbq.org.br, en formato PDF, con acceso gratuito.

\section{REFERENCIAS}

1. In Talanquer, V. (2014). El rol de las suposiciones implícitas y estrategias heurísticas en el razonamiento de los estudiantes de química. Merino, Arellanos y Adúriz-Bravo.(Eds.), Avances en la didáctica de la química, modelos y lenguaje, 93-105.

2. Furió, C.; Furió, C.; Educ. Quim. 2000, 11, 300.

3. Maluta, J. R.; Quim. Nova 2014, 37, 1244.

4. Adúriz-Bravo, A.; Educ. Quim. 2012, 23, 248.

5. Giere, R. N.; Explaining Science: A Cognitive Approach; Science and Its Conceptual Foundations series; University of Chicago Press: Chicago, 1988.

6. Amador, R. Y.; Quintero, N. O.; Adúriz-Bravo, A.; Entre Ciencia e Ingeniería 2018, 12, 116.

7. Develaki, M.; Sci. Educ. 2007, 16, 725.

8. Izquierdo-Aymerich, M.; Adúriz-Bravo, A.; Sci. Educ. 2003, 12, 27.

9. Koponen, I. T.; Sci. Educ. 2007, 16, 751.

10. Reinisch, B.; Krüger, D.; Res. Sci. Educ. 2018, 48, 71.

11. Adúriz-Bravo, A.; Izquierdo Aymerich, M.; Revista Electrónica de Investigación en Educación en Ciencias 2009, 4, 40.

12. Oliva, J. M.; Enseñanza de las Ciencias 2019, 37, 5.

13. Gilbert, J. K.; Int. J. Sci. Math. Educ. 2004, 2, 115.
14. Prins, G. T.; Bulte, A. M. W.; Van Driel, J. H.; Pilot, A.; Res. Sci. Educ. 2009, 39, 681 .

15. Mut, P. N.; Bahamonde, N.; Lozano, E. E.; Cremer, M. C.; TED 2018 , $17,564$.

16. Gilbert, J. K.; Boulter, C. J.; Rutherford, M.; Developing Models in Science Education; Springer Netherlands: Dordrecht, 2000; pp. 193-208.

17. Martínez Díaz, A.; García Rodríguez, M. S.; Suárez Menéndez, J. J.; Enseñanza \& Teaching 2017, 35, 109.

18. Magwilang, E.; Int. J. Learn. Teach. Educ. Res. 2016, 15, 60.

19. Marques, C. M. C.; Lopes, J. B.; Carvalho, M. J.; Quim. Nova 2016, 39, 1254.

20. Lopes, R. M.; Silva Filho, M. V.; Marsden, M.; Alves, N. G.; Quim. Nova 2011, 34, 1275.

21. Wood, D.; Bruner, J. S.; Ross, G.; Journal of Child Psychology and Psychiatry 1976, 17, 89.

22. Hannafin, M.; Land, S.; Oliver, K.; Open learning environments: Foundations, methods, and models; Springer: New York, 1999; Vol. 2.

23. Koli, A. R.; Ranch, K. M.; Patel, H. P.; Parikh, R. K.; Shah, D. O.; Maulvi, F. A.; Int. J. Pharm. 2021, 596, 120202.

24. He, Y.; Zhan, C.; Pi, C.; Zuo, Y.; Yang, S.; Hu, M.; Bai, Y.; Zhao, L.; Wei, Y.; AAPS PharmSciTech 2020, 21, 170.

25. Eltze, M.; Sanders, K. H.; Boss, H.; Boer, R.; Ulrich, W. -R; Flockerzi, D.; Chirality 1990, 2, 233.

26. Schoch, G. A.; Yano, J. K.; Sansen, S.; Dansette, P. M.; Stout, C. D.; Johnson, E. F.; J. Biol. Chem. 2008, 283, 17227.

27. Atkins, P.; de Paula, J.; Physical Chemistry: Thermodynamics, Structure, and Change; WH Freeman and Company: New York, 2014.

28. Taylor, S. J.; Bogdan, R.; DeVault, M.; Introduction to qualitative research methods: A guidebook and resource; John Wiley \& Sons: Hoboken, 2015.

29. Okuda Benavides, M.; Gómez-Restrepo, C.; Revista Colombiana de Psiquiatría 2005, 34, 118. 